\title{
Expression of miR-195 is associated with chemotherapy sensitivity of cisplatin and clinical prognosis in gastric cancer
}

\author{
Rui $\mathrm{Ye}^{1,2, *}$, Bo $\mathrm{Wei}^{3, *}$, Sheng $\mathrm{Li}^{4, *}$, Wei Liu ${ }^{5, *}$, Juntao Liu', Luan Qiu ${ }^{2}$, Xuan Wu ${ }^{2}$, \\ Zhifei Zhao ${ }^{2}$ and Jianxiong $\mathbf{L i}^{\mathbf{7}}$ \\ ${ }^{1}$ Department of Oncology, Beidaihe Sanatorium of Beijing Military Command, Qinhuangdao 066100, Hebei, P.R. China \\ ${ }^{2}$ Department of Radiotherapy, Chinese PLA General Hospital, Beijing 100853, P.R. China \\ ${ }^{3}$ Department of General Surgery, Chinese PLA General Hospital, Beijing 100853, P.R. China \\ ${ }^{4}$ Department of Biological Repositories, Zhongnan Hospital of Wuhan University, Wuhan 430071, Hubei, P.R. China \\ ${ }^{5}$ Institute of Microbiology and Epidemiology, Academy of Military Medical Sciences, Beijing 100853, P.R. China \\ ${ }^{6}$ Department of General Thoracic Surgery, Affiliated Hospital of Logistics University of Chinese People's Armed Police Forces, \\ Tianjin 300162, P.R. China \\ ${ }^{7}$ Department of Radiotherapy, Hainan Branch of Chinese PLA General Hospital, Sanya 572000, Hainan, P.R. China \\ *These authors have contributed equally to this work
}

Correspondence to: Jianxiong Li, email: jianxiongli519@163.com

Keywords: miR-195; AKT3; gastric cancer; chemotherapy sensitivity

Received: May 27, $2017 \quad$ Accepted: September 21, $2017 \quad$ Published: October 19, 2017

Copyright: Ye et al. This is an open-access article distributed under the terms of the Creative Commons Attribution License 3.0 (CC BY 3.0), which permits unrestricted use, distribution, and reproduction in any medium, provided the original author and source are credited.

\section{ABSTRACT}

Gastric cancer has higher morbidity and mortality than other cancers for the low diagnosis rate and few therapies. MiR-195 has been reported to be involved in the occurrence, development and prognosis of various cancers. However, the function of miR-195 in gastric cancer remains largely unknown. Herein, the aims of this study were to probe the functional mechanism of miR-195 and its chemotherapy sensitivity as well as clinical prognosis in gastric cancer. We screened out low-expressed miR195 through microarray analysis and further confirmed miR-195 was widely downregulated in gastric cancer cells. Subsequently, AKT3 was identified as the direct target gene of miR-195 by target gene prediction software, dual luciferase reporter assay and western blot. Functional assays indicated that miR-195 acted as a tumor suppressor through regulating the proliferative, migrated and invasive properties of gastric cancer cells in vitro, and intratumoral delivery of miR-195 significantly suppressed tumor growth in vivo. Additionally, we also found miR-195 overexpression could enhance the chemotherapy sensitivity of cisplatin in gastric cancer cells and prolong the overall survival and progression free survival of gastric cancer patients. Collectively, our findings demonstrate miR-195 may be of great significance on early diagnosis of gastric cancer, providing the theoretical basis for prognosis and recurrence risk.

\section{INTRODUCTION}

MicroRNAs (miRNAs) are endogenous noncoding regulatory RNAs with 17-25 nucleotides, which play vital roles in post-transcriptional gene regulation [1]. Up to now, more than 2,000 miRNAs have been registered in the "miRBase" database [2]. MiRNAs are strongly linked with biological processes consisting of cell proliferation, metastasis, differentiation, and apoptosis [3, 4]. Mounting evidence has demonstrated that multiple miRNAs are abnormally expressed in human cancers and functionally participate in regulation of various pathological processes 
during tumor initiation and progression [5]. Several miRNAs have been identified as either oncogenes or tumor suppressors. For example, miR-195 as a tumor-suppressive miRNA is correlated with metastases and recurrences of gastric cancer (GC) [6], breast cancer [7], and human cervical cancer [8], while miR-21 as a well-characterized oncogenic miRNA is overexpressed in breast cancer, colorectal cancer, lung cancer, GC and so on [9-12].

$\mathrm{GC}$ is one of the most common malignancies with a high mortality rate that ranks as the second leading cause of cancer-related deaths worldwide [13]. Available therapeutic methods are limited due to the fact that the majority of GC patients are diagnosed at advanced stages. Currently, surgery remains the main curative therapy, while perioperative and adjuvant chemotherapy with chemotherapy drugs such as fluoropyimidine (5-FU commonly used), platinum (cisplatin (DDP) commonly used), and antharcycline (doxorubicin commonly used), can improve outcome of resectable GC [14]. Over the past few years, great progresses in the diagnosis and treatment of GC have been made. However, the 5-year-survival rate in those patients is still unsatisfactory on account of its recurrence and metastasis $[15,16]$. Just as mentioned above, miR-195 as a tumor-suppressive miRNA is associated with metastasis and recurrence of GC [8]. As the role of miR-195 in tumorigenesis is gradually discovered, the great significance of this molecule associated with clinical diagnosis and prognosis has been attracting more and more attentions from scholars.

The serine/threonine kinase AKT, a major downstream mediator of the phosphoinositide 3-kinase (PI3K)-pathway, is known to be related with disease free survival and tumor progression $[17,18]$. AKT3 is one of the three highly homologous AKT isoforms, which contributes to the formation of metastases. Nowadays, emerging data have indicated that AKT3 is also strongly linked with several other malignancies, including GC, ovarian cancer, glioblastoma, breast cancer, triple negative breast cancer, and hepatocellular carcinoma [19-23].

In the present study, the functional role of miR195 in GC progression was firstly evaluated in vitro and in vivo, and then its relationship with chemotherapy sensitivity and clinical prognosis was analyzed.

\section{RESULTS}

\section{MiR-195 was remarkably down-regulated in GC samples}

Two tumor tissues and adjacent tissues were used to fulfill microarray analysis and fold change method was employed to conduct differential analysis of miRNA expression levels in the two group tissues. Clear-cut distinction was drawn in the sixteen miRNA expression levels (Figure 1A, fold change $>2$ ). MiR-195 attained our focus from these significantly differentially expressed
miRNAs for the fact that miR-195 expression was evidently down-regulated and its relevant research in GC has largely unreported. According to microarray analysis and miRBase, its mature sequence was confirmed, which was described in corresponding Materials and methods part. Subsequently, real-time quantitative polymerase chain reaction (RT-qPCR) was performed on twenty-nine tumor tissues and adjacent tissues to detect the mRNA expression of miR-195. Of those twenty-nine tumor tissues, miR-195 expression was downregulated in twenty-one tissues (Figure 1B), accounting for $72.4 \%$. In addition, the statistical analysis revealed an evident statistical significance in the relative expression level of miR-195 between tumor tissues and adjacent tissues (Figure $1 \mathrm{C}, P<0.001$ ).

\section{AKT3 was a direct target of miR-195}

Since the fact that miRNAs exert their molecular functions by mediating the target genes thereof, we explored the candidate target genes of miR-195 using Targetscan, MIRanda and PicTar. Ten putative target genes, consisting of CD28, BCL2L2, MAP2K1, KLC2, NRAS, HOXA10, HOXA3, BCL9L, STX1A, AKT3, were primarily selected according to dual luciferase reporter assays (Supplementary Figure 1). In concrete, taking AKT3 for instance, pMIRAKT3-WT and pMIR-AKT3-Mut (Figure 2A) were constructed. Afterwards, the pRL-TK vector with pGL3 vector, pMIR-AKT3-WT vector or pMIR-AKT3-Mut vector were in the combination of miR-195 mimic or scramble (Scr) to co-transfect $293 \mathrm{~T}$ cells. The results suggested that the relative luciferase activity was evidently suppressed by miR-195 overexpression in cells transfected with pMIR-AKT3-WT $(P<0.001)$, while it showed no difference of luciferase activity in cells transfected with pMIR-AKT3-Mut (Figure 2B). So were the cases in BCL2L2 (Supplementary Figure 1, $P<0.01$ ) and NRAS (Supplementary Figure 1, $P<0.05$ ). These results covered that AKT3, BCL2L2 and NRAS were likely to be direct targets of miR-195 in GC. Subsequently, primary RT-qPCR assay revealed that AKT3 mRNA activity was significantly suppressed in miR-195 mimic group (Supplementary Figure $2, P<0.001$ ), while no clear-cut distinction was drawn among BCL2L2 group and NRAS group (Supplementary Figure 2). It was therefore AKT3 carried the first possibility to be the direct target of miR-195. To further verify this finding, miR-195 mimic or Scr was transfected into GC cell lines HGC-27 and MGC-803, respectively. Western blot showed that the AKT3 protein expression was sufficiently decreased in miR-195 mimic group when compared with Scr group $(P<0.001)$, whereas it showed no difference between Scr group and untreated group at AKT3 protein expression level (Figure 2C). Similar to primary RT-qPCR assay, the obtained results indicated that the AKT3 mRNA expression in miR-195 mimic group was significantly lower than that in Scr group (Figure 2D, $P<0.001$ ), certifying that AKT3 was a direct target of miR-195 in GC. 


\section{MiR-195 inhibited cell proliferation, migration and invasion in vitro GC cells}

To investigate the functional roles of miR-195 in $\mathrm{GC}$, cell proliferation, migration, and invasion assays were conducted in HGC-27 and MGC-803 cells transfected with miR-195 mimic or Scr. Cell Counting Kit-8 (CCK8 ) assays revealed that the proliferative abilities of HGC27 and MGC-803 cells transfected with miR-195 mimic were significantly suppressed compared with those transfected with the Scr (Figure 3A, $P<0.05$ ). Similarly, we found that both the migrated and invasive properties of cells transfected with miR-195 mimic were remarkably impaired when compared with those transfected with the Scr via scratch wound-healing assays and transwell assays (Figure 3B, 3C, $P<0.001$ ).

\section{MiR-195 showed a visible inhibitory impact on tumor growth in vivo}

In this section, the impact of miR-195 overexpression on $\mathrm{GC}$ progression in vivo was examined using intratumoral delivery approach. MGC-803 cells were injected subcutaneously into nude mice, and then miR-195 agomir
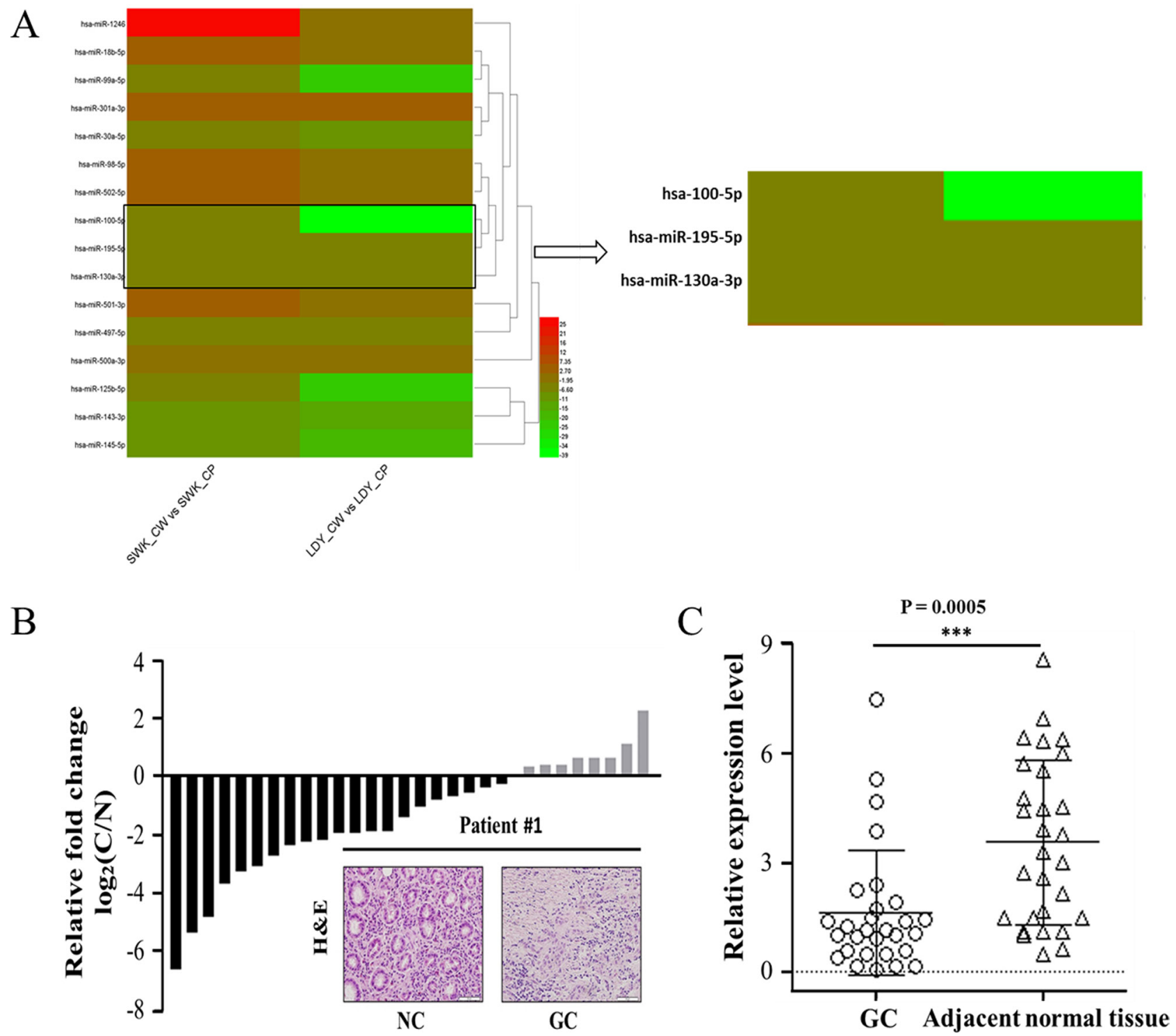

Figure 1: MiR-195 was remarkably down-regulated in GC samples. (A) Heat maps of differentially expressed miRNAs between GC tissues and adjacent normal tissues. LDY_CP, LDY_CW, SWK_CP and SWK_CW are all sample IDs, wherein CP represents adjacent normal tissues and CW refers to GC tissues. Red represents higher expression and green represents lower expression. (B) RT-qPCR analysis of miR-195 expression in twenty-nine pairs of GC tissues and H\&E stain of GC and adjacent tissues of patient\#1. (C) The statistical analysis of miR-195 relative expression level in tumor tissues and adjacent tissues. Horizontal line represents the average value for each group. NC, negative control; GC, gastric cancer; RT-qPCR, real-time quantitative polymerase chain reaction; ${ }^{* * *}, P<0.001$ 
or negative control (NC) was injected on the $10^{\text {th }}$ day. Tumor volume was calculated every four days. The results indicated that tumor volumes presented a slower growth in miR-195-treated group than in NC group (Figure 4A, $P<0.05)$. The mice were killed due to the presence of large tumor in $\mathrm{NC}$ group on the $26^{\text {th }}$ day. Representative photograph of xenograft tumors showed that tumor sizes in miR-195-treated group were significant smaller than those in NC group (Figure 4B). Meanwhile, we observed that tumor weight increased evidently in NC group when compared with that in miR-195-treated group (Figure 4C, $P$ $<0.05)$. In addition, RT-qPCR of xenograft tumors revealed that miR-195 expression was up-regulated in miR-195treated group (Figure 4D, $P<0.05$ ). Taken together, these findings verified that miR-195 overexpression significantly suppressed tumor growth in vivo.

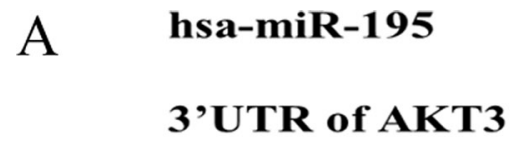

hsa-miR-195

3'UTR of AKT3
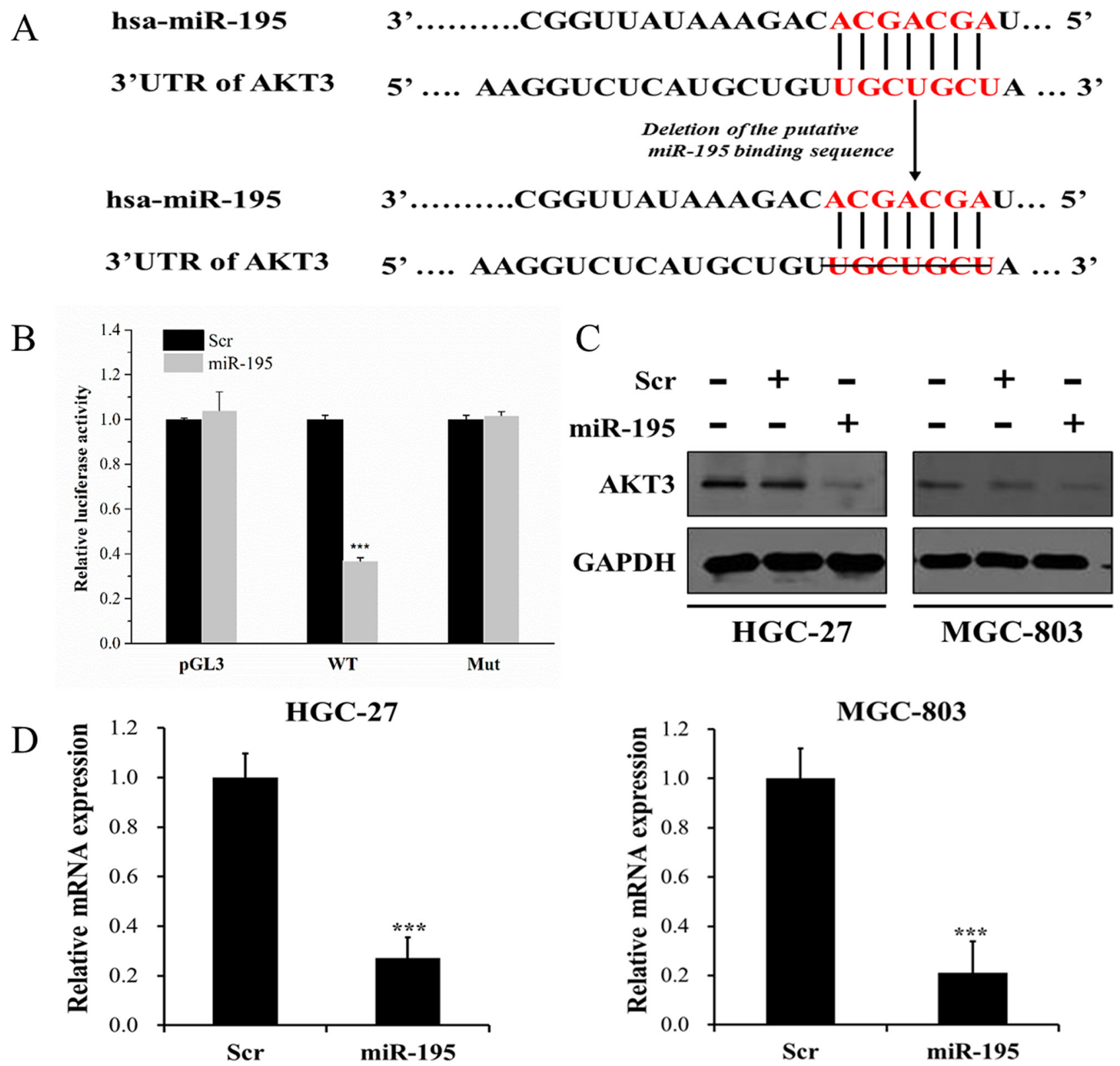

Figure 2: AKT3 was identified as a direct target of miR-195. (A) Schematic representation of AKT3 3'-UTR showing putative miR-195 target sites. (B) The results suggested that the relative luciferase activity was evidently suppressed by miR-195 overexpression in cells transfected with pMIR-AKT3-WT, while it showed no difference of luciferase activity in cells transfected with pMIR-AKT3-Mut. (C) Western blot showed that the AKT3 protein expression was sufficiently decreased in miR-195 mimic group when compared with Scr group, whereas it showed no difference between Scr group and untreated group at AKT3 protein expression level. (D) The RT-qPCR assay indicated that the AKT3 mRNA expression in miR-195-treated group was significantly lower than that in Scr group. Error bars represented standard deviation obtained from three independent experiments and all the data were shown as mean \pm SD. WT, wild type; Mut, mutation; Scr, scramble; RT-qPCR, real-time quantitative polymerase chain reaction; ${ }^{* * *}, P<0.001$ 


\section{Overexpression of miR-195 enhanced the chemotherapy sensitivity of DDP in GC cells}

To discover the influence of DDP concentration on the chemotherapy sensitivity of miR-195 in GC cells, we transfected miR-195 mimic or Scr into HGC-27 and MGC-803 cells. Afterwards, different concentrations of DDP (i.e., $0,10,20,40,60$ and $80 \mu \mathrm{mol} / \mathrm{L}$ ) were added into each group. Then, the viability of those two cell lines treated with different concentrations of DDP was evaluated using CCK-8 assays. As depicted in Figure 5A, overexpression of miR-195 remarkably impaired the GC cell viability at each concentration of DDP when compared with the Scr (Figure 5A, $P<0.01$ ), indicating that miR195 could enhance the chemotherapy sensitivity of DDP at different concentration in GC cells. In addition, we also noticed that the IC50 value of DDP was $50 \mu \mathrm{mol} / \mathrm{L}$.
To further determine the influence of functional time on the chemotherapy sensitivity of miR-195 for DDP in GC cells, CCK-8 assays were respectively conducted after adding $50 \mu \mathrm{mol} / \mathrm{L}$ (IC50) DDP for varied times (i.e., $0 \mathrm{~h}$, $12 \mathrm{~h}, 24 \mathrm{~h}, 36 \mathrm{~h}$ and $48 \mathrm{~h}$ ). Similarly, we found that miR195 could enhance the chemotherapy sensitivity of DDP at varied times in GC cells (Figure 5B, $P<0.05$ ).

\section{The correlation analyses between miR-195 expression and clinical manifestation and prognosis of patients with GC}

To examine the correlation between miR-195 and clinicopathological characteristics of patients with GC, the clinical data of twenty-nine patients were collected (Table 1), including gender, age, vascular invasion, tumor location, Borrmann type, tumor node metastasis
$\mathbf{A}$

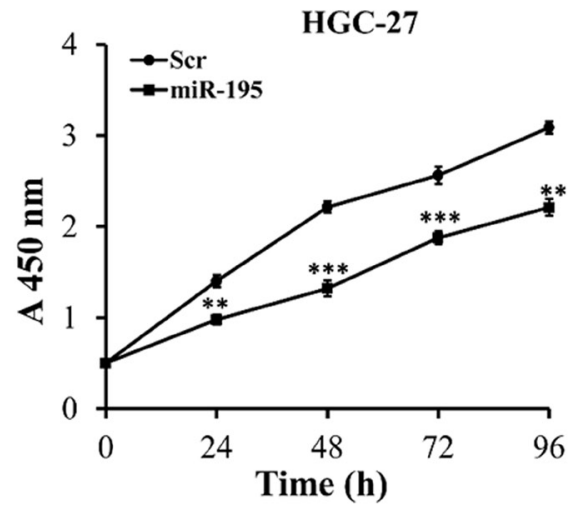

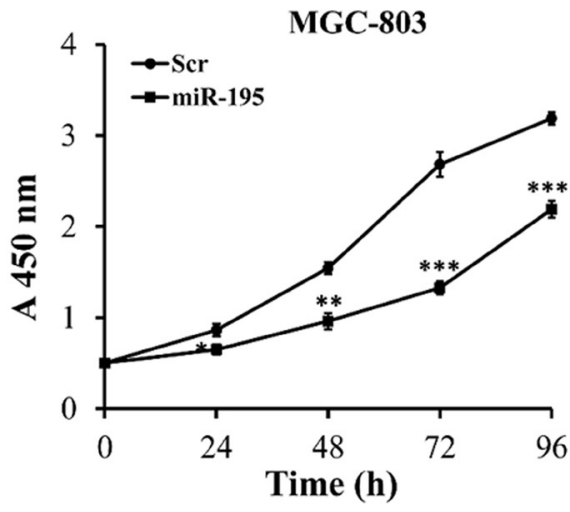

B

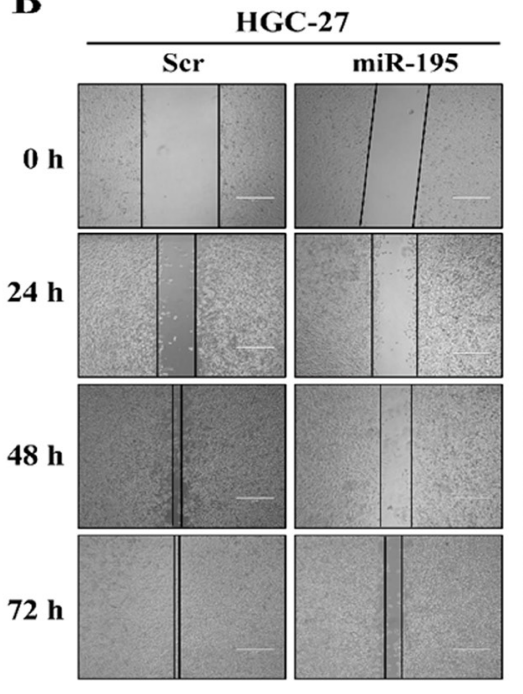

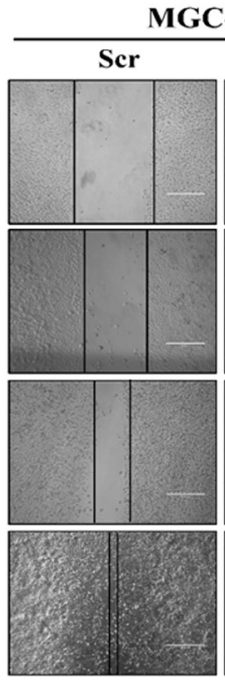

MGC-803

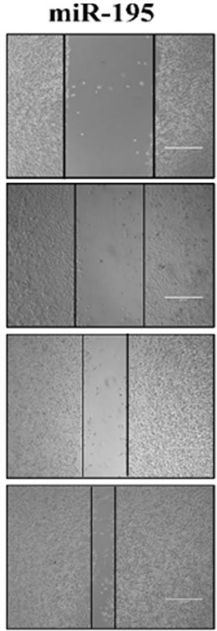

C

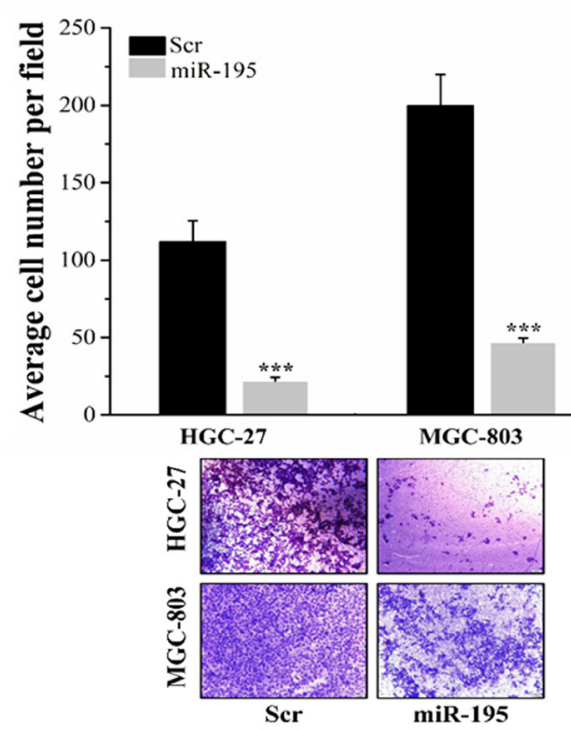

Figure 3: MiR-195 inhibited cell proliferation, migration and invasion in vitro GC cells. (A) CCK-8 assays revealed that the proliferative abilities of HGC-27 and MGC-803 cells transfected with miR-195 mimic were significantly suppressed compared with those transfected with the Scr. Scratch wound-healing assays (B) and transwell assays (C) revealed that both the migrated and invasive properties of cells transfected with miR-195 mimic were remarkably impaired when compared with those transfected with the Scr. Error bars represented standard deviation obtained from three independent experiments and all the data were shown as mean $\pm \mathrm{SD}$. All the scale bars are $500 \mu \mathrm{m}$. GC, gastric cancer; CCK-8, Cell Counting Kit-8; Scr, scramble; ${ }^{*}, P<0.05 ;{ }^{* *}, P<0.01 ;{ }^{* * *}, P<0.001$ 
(TNM) stage and grade. No clear-cut correlation was observed between miR-195 expression and above clinicopathological features. So was the case in the AKT3 expression (Supplementary Table 1). Furthermore, we performed the correlation of miR-195 expression with overall survival (OS), and progression free survival (PFS). The results indicated that the median survival time (MST) of OS for miR-195 high-expression patients was over sixty months and for low-expression was about twenty-two months (Figure 6A, $P=0.023$ ), while the corresponding MST of PFS was respectively more than sixty months and around eleven months (Figure 6B, $P=0.010$ ). Taken together, it could be concluded that miR-195 expression was related with both OS and PFS.

\section{DISCUSSION}

Recently, numerous evidences have demonstrated that miRNAs expression has significant differences in
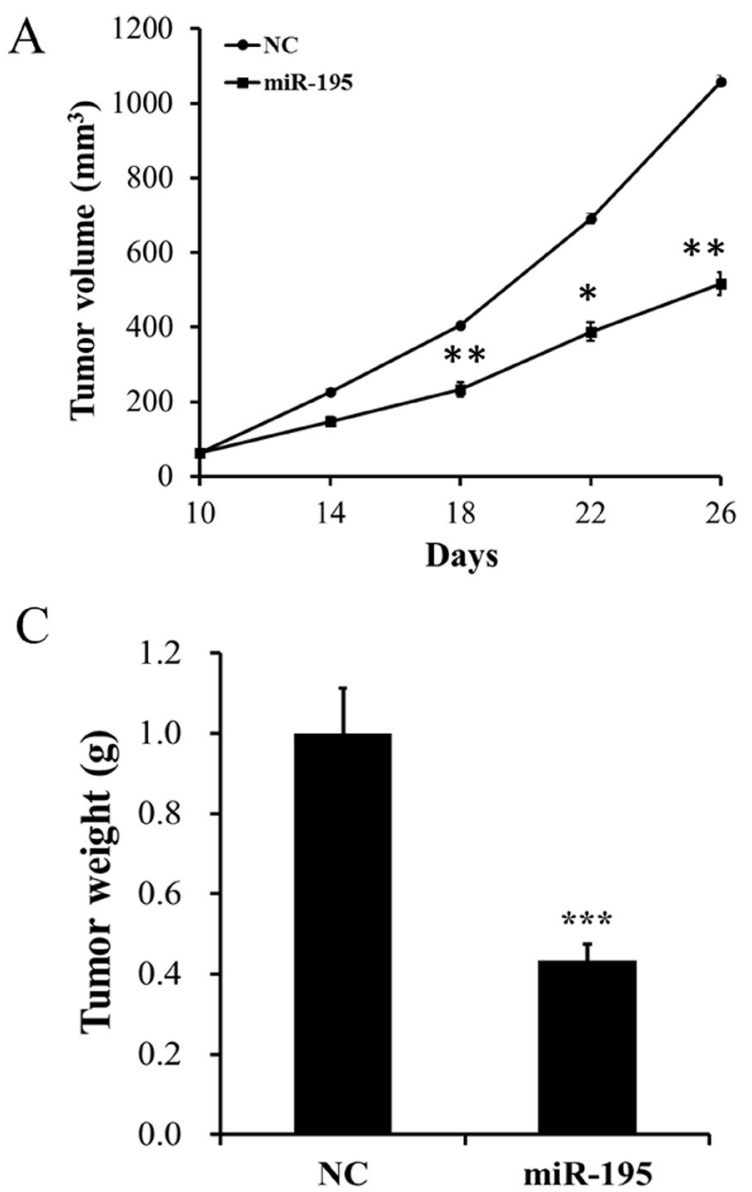

tumor tissues and normal tissues. Those differentially expressed miRNAs are frequently located at fragile sites which are generally implicated in cancer occurrence [5]. Meanwhile, plentiful studies have proved that that miRNAs exist in the variable genome involved in cancers, which makes researchers focus on the role of miRNAs in the process of cancer development [24]. For example, the expression profile of miR-21 was identified by miRNA microarray analysis and its expression was obviously up-regulated in lung cancer tissues, exhibiting a high association with tumorigenesis and various prognosis factors [11, 25]. Another example is that twenty nine miRNAs were identified to be expressed differentially in breast cancer cells, and more importantly, those ectopic expression miRNAs could up-regulate or down-regulate their target genes to down-regulate the anti-apoptotic gene expression, contributing to the apoptosis of breast cancer cells $[26,27]$.
B

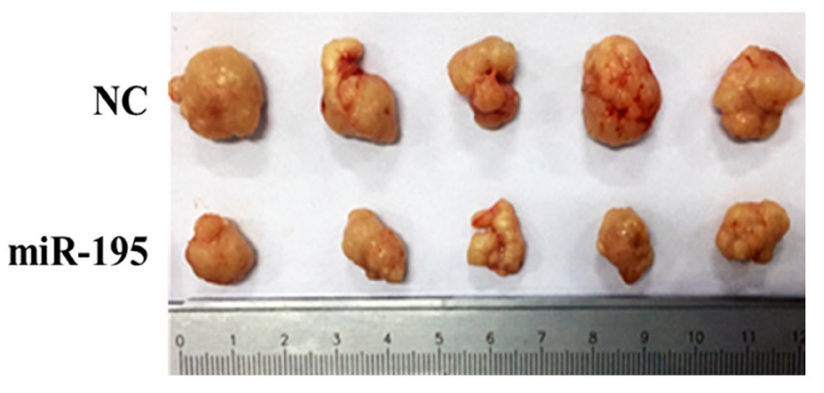

$\mathrm{D}$

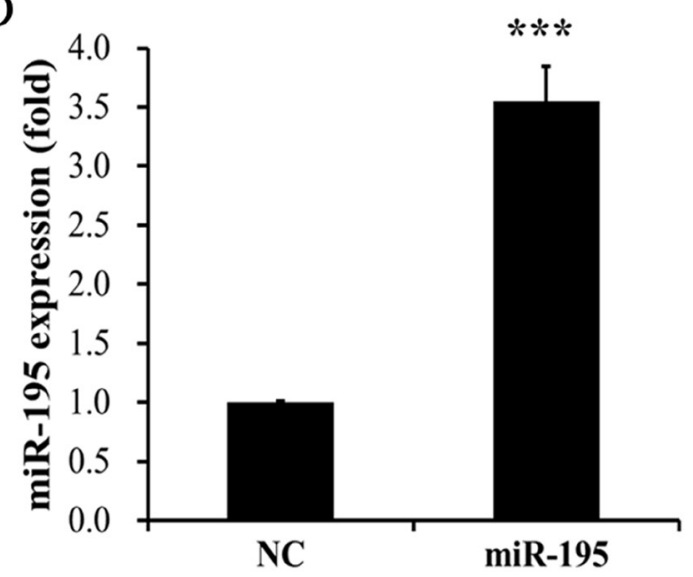

Figure 4: MiR-195 showed a visible inhibitory impact on tumor growth in vivo. (A) Growth curve of tumor volumes presented a slower growth in miR-195-treated group than those in NC group. The first intratumor injection of miR-195 was performed once palpable tumors (average volume of $62.5 \mathrm{~mm}^{3}$ ) were detected (usually on the 10th day). (B) Representative photograph of xenograft tumors showed that tumor size in miR-195-treated group was significant larger than that in NC group. (C) Tumor weight increased evidently in NC group when compared with that in miR-195-treated group. (D) RT-qPCR of xenograft tumors revealed that miR-195 expression was up-regulated in miR-195-treated group. NC, negative control; RT-qPCR, real-time quantitative polymerase chain reaction; ${ }^{*}, \mathrm{P}<0.05 ;{ }^{* *}, \mathrm{P}<0.01 ;{ }^{* * *}$, $\mathrm{P}<0.001$ 
Previous studies have deeply investigated the regulation mechanisms of miR-195 in a variety of common cancers, such as hepatocellular carcinoma [28], colorectal cancer [29], non-small cell lung cancer [30] and breast cancer $[6,31]$. However, the research about the regulation mechanisms of miR-195 in GC is just emerging. It has been verified that miR-195 expression was down-regulated in GC tissue when compared with its corresponding paracancerous tissue [32]. Meanwhile, some studies have shown the methylation modulation may be one of the mechanisms inducing the decreased expression of miR-195, who may play a role in tumor inhibition by regulating CDK6 kinase and vascular endothelial growth factor (VEGF) [8]. Despite of abundant investigations on the relationship between miR-195 expression and GC, the specific regulatory mechanism of miR-195 in GC has not been further explored, and the target gene of miR-195 has not been clearly validated. Therefore, this study was performed to uncover the specific regulatory mechanism of miR-195 in GC.

In present study, we first screened out low-expressed miR-195 based on the microarray analysis and confirmed that miR-195 was widely down-regulated in GC cells by RT-qPCR assays, which were consistent with previous studies [8, 32]. Additionally, a series of functional assays indicated that miR-195 was regarded as a tumor suppressor through regulating the proliferative, migrated and invasive properties of GC cells in vitro. The in vivo
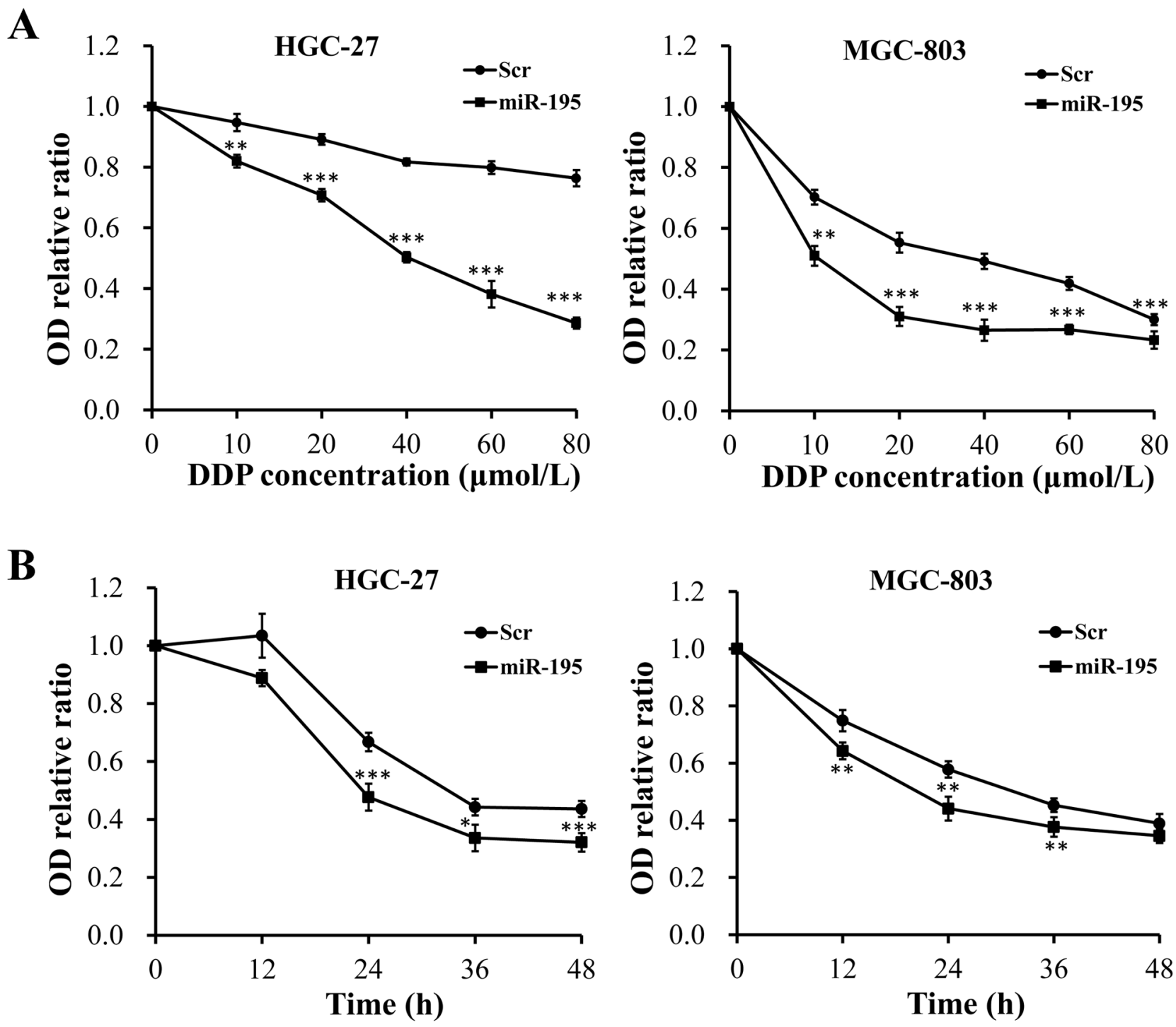

Figure 5: Overexpression of miR-195 enhanced the chemotherapy sensitivity of DDP in GC cells. (A) CCK-8 assays indicated that overexpression of miR-195 remarkably impaired the GC cell viability at each concentration of DDP when compared with the Scr. (B) CCK-8 assays showed that miR-195 could enhance the chemotherapy sensitivity of DDP at varied times in GC cells. Error bars represented standard deviation obtained from three independent experiments and all the data were shown as mean \pm SD. DDP, cisplatin; GC, gastric cancer; CCK-8, Cell Counting Kit-8; Scr, scramble; ${ }^{*}, P<0.05 ;{ }^{* *}, P<0.01 ;{ }^{* * *}, P<0.001$ 
intratumoral delivery of miR-195 into subcutaneous xenografts significantly alleviated tumor growth. The miR-195 expression was obviously higher in miR-195treated tumors (fold change $>2$ ) than in NC tumors, highlighting the role of miR-195 in tumor progression in vivo. Generally, the biological functions of miRNAs were determined by their downstream target genes. Hence, three prediction softwares (Targetscan, MIRanda and PicTar) were used to screen the direct target gene of miR-195. Ten putative target genes, consisting of CD28, BCL2L2, MAP2K1, KLC2, NRAS, HOXA10, HOXA3, BCL9L, STX1A, AKT3, were primarily selected according to dual luciferase reporter assays and RT-qPCR assay. Ultimately, AKT3 was verified to be the direct target of miR-195 via dual luciferase reporter assays, Western blot coupled with RT-qPCR assay. It is well-known that AKT3 is an important member of PI3K/AKT signaling pathway [33].

In recent studies, it has been suggested that PI3K/ AKT signaling pathway plays a significant role in the occurrence and development of multiple cancers, including lung cancer, GC, as well as head and neck cancer [34]. However, the correlation between AKT3 and miRNAs has rarely been reported. In the present paper, we affirmed that AKT3 was a direct target gene of miR-195 for the first time and speculated that miR-195 was likely to inhibit the proliferative, migrated and invasive properties of GC cells through PI3K/AKT signaling pathway.

Recently, emerging evidences have shown that miR195 can enhance the chemotherapy sensitivity in various cancers. For example, it has been reported that miR-195 plays a positive effect on the chemotherapy sensitivity of doxorubicin in colon cancer cells by targeting the first binding site of BCL2L2 mRNA [35]. Another study about that miR195 increases the chemotherapy sensitivity of 5-FU in human hepatocellular carcinoma cells by targeting BCL-w has also been reported [36]. However, the study concerning the effect of miR-195 on chemotherapy sensitivity in GC has not been reported up to now. Yan B et al. has confirmed that miRNA$29 \mathrm{~b}$ can increase the chemotherapy sensitivity of DDP in prostate cancer cells by targeting AKT3 [37]. In this study, we confirmed that AKT3 was the direct target gene for miR195 in GC cells. We were therefore tempting to speculate that miR-195 may be linked to chemotherapy sensitivity of DDP. Accordingly, related experiments were performed and the obtained results revealed that miR-195 could enhance the chemotherapy sensitivity of DDP in GC cell lines at different times. We conclude that miR-195 has a promoting effect on chemotherapy sensitivity in GC cells, which provides a theoretical and experimental basis for the exploration of miR-195 new targets. Additionally, the relationship between miR-195 and the OS and PSF of postoperative patients with $\mathrm{GC}$ also has not been illustrated. Hereby, we performed the correlation analysis between miR-195 expression and clinical data. The results showed that miR-195 expression was remarkably associated with the OS and PSF of postoperative patients with GC and the OS of patients with high-expressed miR-195 was evidently prolonged when compared with lowexpressed group.

In conclusion, these data are consistent with the possibility that miR-195 plays a critical functional role in
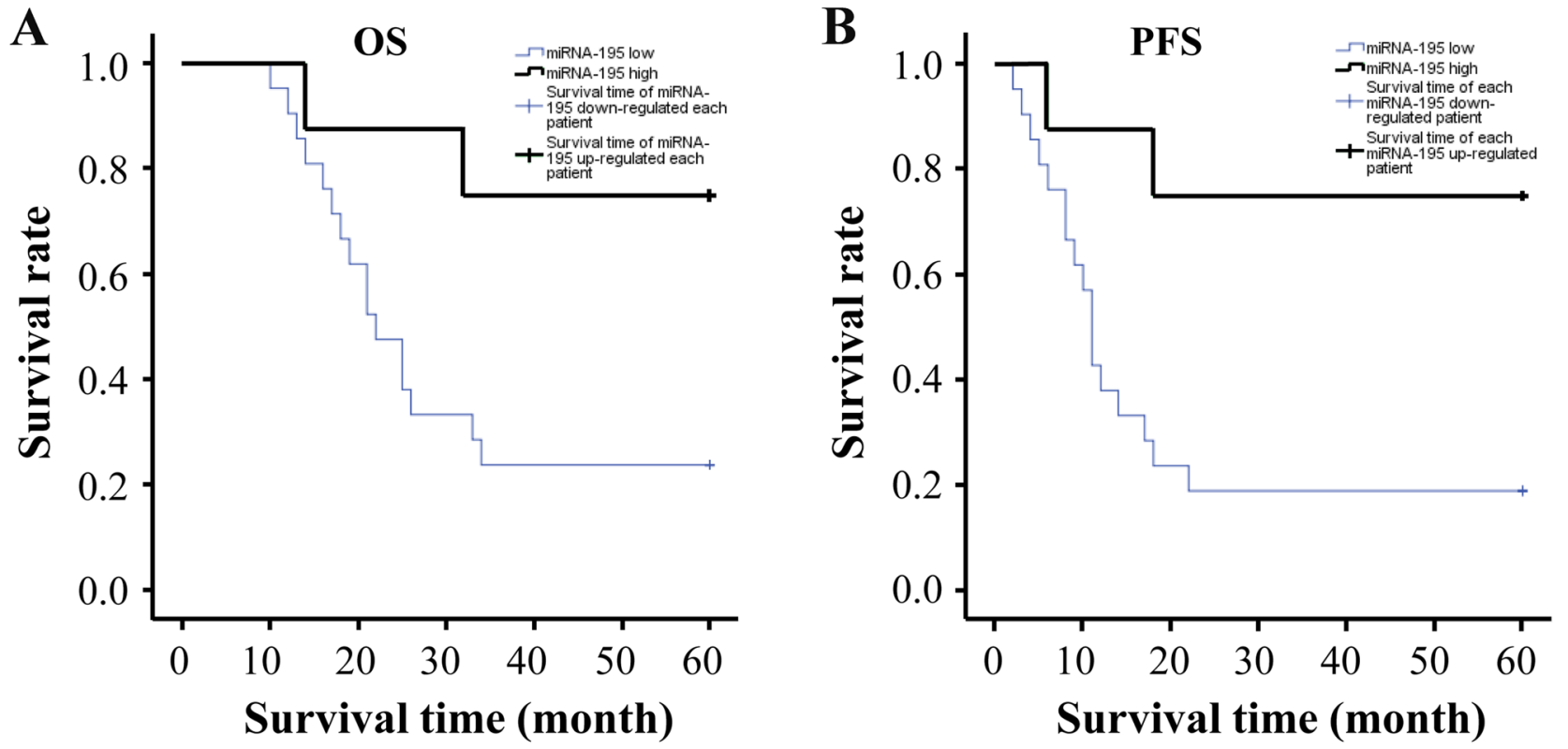

Figure 6: The correlation analyses between miR-195 expression and prognosis of patients with GC. The results indicated that (A) the MST of OS for miR-195 high-expression patients was over sixty months and for low-expression was about twenty-two months $(P=0.023)$, while $(\mathbf{B})$ the corresponding MST of PFS was more than sixty months and around eleven months, respectively $(P=0.010)$. MST, median survival time; OS, overall survival; PFS, progression free survival 
Table 1: Correlation analysis between miR-195 expression in GC and clinicopathological characteristics

\begin{tabular}{|c|c|c|c|c|c|}
\hline \multicolumn{2}{|c|}{ Clinicopathological characteristics } & \multirow{2}{*}{$\frac{\text { Number of cases }}{22}$} & \multirow{2}{*}{$\begin{array}{c}\text { Mean }^{1} \\
-1.718\end{array}$} & \multirow{2}{*}{$\frac{\mathbf{S D}^{2}}{2.202}$} & \multirow{3}{*}{$\begin{array}{c}\text { P-value } \\
0.181\end{array}$} \\
\hline \multirow{2}{*}{ Gender } & Male & & & & \\
\hline & Female & 7 & -0.769 & 1.305 & \\
\hline \multirow{2}{*}{ Age } & $\leq 60$ & 16 & -1.567 & 2.264 & \multirow{2}{*}{0.820} \\
\hline & $>60$ & 13 & -1.393 & 1.820 & \\
\hline \multirow{2}{*}{ Vascular invasion } & Positive & 15 & -0.971 & 1.736 & \multirow{2}{*}{0.166} \\
\hline & Negative & 14 & -2.044 & 2.259 & \\
\hline \multirow{3}{*}{ Tumor location } & Cardia & 5 & -3.011 & 2.202 & \\
\hline & Gastric body & 3 & 0.137 & 1.889 & \\
\hline & Gastric antrum & 21 & -1.359 & 1.877 & \\
\hline \multirow{2}{*}{ Borrmann type } & $\mathrm{I}+\mathrm{II}$ & 15 & -1.559 & 1.693 & \multirow{2}{*}{0.854} \\
\hline & $\mathrm{III}+\mathrm{IV}$ & 14 & -1.414 & \multirow[t]{2}{*}{2.427} & \\
\hline \multirow{2}{*}{ pT stage } & $\mathrm{T} 1+\mathrm{T} 2$ & 1 & -2.374 & & \multirow{4}{*}{0.581} \\
\hline & $\mathrm{T} 3+\mathrm{T} 4$ & 28 & -1.457 & & \\
\hline \multirow{2}{*}{ pN stage } & $\mathrm{N} 0+\mathrm{N} 1$ & 12 & -1.245 & 1.743 & \\
\hline & $\mathrm{N} 2+\mathrm{N} 3$ & 17 & -1.662 & 2.267 & \\
\hline \multirow{2}{*}{ pM stage } & M0 & 28 & -1.563 & & \\
\hline & M1 & 1 & 0.591 & & \\
\hline \multirow{2}{*}{ pTNM stage } & $\mathrm{I}+\mathrm{II}$ & 6 & -1.204 & 2.079 & \multirow{2}{*}{0.716} \\
\hline & $\mathrm{III}+\mathrm{IV}$ & 23 & -1.563 & 2.074 & \\
\hline \multirow{2}{*}{ Histologic grade } & well & 10 & -2.500 & 2.577 & \multirow{2}{*}{0.106} \\
\hline & poor & 19 & -0.957 & 1.519 & \\
\hline
\end{tabular}

${ }^{1}$ Mean of $\log _{2}(\mathrm{C} / \mathrm{N}) ;{ }^{2}$ Standard Definition of $\log _{2}(\mathrm{C} / \mathrm{N})$; C: normalized expression of cancer tissues; N: normalized expression of adjacent noncancerous tissues.

suppressing tumor progression in human GC in vitro and in vivo, probably exerting its role by modulating its target gene AKT3. Meanwhile, miR-195 has a significant effect on the chemotherapy sensitivity and clinical prognosis. Taken together, our findings indicate that miR-195 is promising to act as a therapeutic target for treatment and a biomarker for progression of human GC.

\section{MATERIALS AND METHODS}

\section{Patient samples}

Specimens of both GC tissues and corresponding adjacent normal tissues ( $>5 \mathrm{~cm}$ away from cancer tissues) were collected from 29 patients who received initial surgery in Chinese PLA General Hospital from 2010 to 2011. The utilizations of the tumor samples for research were approved by the ethical committee of Chinese PLA General Hospital prior to collection. In all patients, everyone had provided informed consent and no one had undergone therapy before resection. All collected samples were frozen immediately in liquid nitrogen after resection or kept in a refrigerator at $-80^{\circ} \mathrm{C}$.

\section{Cell culture}

The human GC cell lines HCG-27, MGC-803 and 293T were all purchased from the Cell Centre of Peking Union Medical College Hospital. Cells were cultured in Dulbecco's modified Eagle's medium (DMEM) (HyClone, USA) supplemented with $10 \%$ fetal bovine serum (Hangzhou, China), $100 \mathrm{IU} / \mathrm{ml}$ penicillin and $100 \mu \mathrm{g} / \mathrm{ml}$ streptomycin (Gibco, Invitrogen, Shanghai, China) in a humid atmosphere containing $5 \% \mathrm{CO}_{2}$ at $37^{\circ} \mathrm{C}$.

\section{Plasmid construction and transfection}

Cells were plated into 24-well culture plate at a density of $5 \times 10^{4}$ cells pre well and cultured to attain a concentration of $70 \% \sim 80 \%$ prior 
to transfection. Afterwards, transfection was performed with Lipofectamine 2000 (Invitrogen, Carlsbad, CA, USA) following the manufacturer's instruction. The sequences used in this part were presented as follows: hsa-miR-195 mimic, 5'-UAGCAGCACAGAAAUAUUGGC-3' (sense); NC miRNA mimic, 5'-UUCUCCGAACGUGUCACGUTT-3' (sense). At $24 \mathrm{~h}$ after transfection, the cells were harvested for further functional assays.

\section{RNA extraction and real-time quantitative polymerase chain reaction (RT-qPCR)}

According to the manufacturer's protocol, total RNA was isolated from GC cell lines using Trizol reagent (Invitrogen, Carlsbad, CA, USA). To detect mRNA, first-strand cDNA was synthesized by PrimeScript RT reagent kit (TaKaRa, Dalian, China). RT-qPCR was then performed using the ABI PRISM 7500 Fast RealTime PCR System (Perkin Elmer/Applied Biosystems, Rotkreuz, Switzerland) with SYBR Premix Ex Taq ${ }^{\text {TM II }}$ (Takara) according to the manufacturer's instructions. U6 snRNA was used as an internal control to normalize the experimental results. As for miRNA detection, cDNA is reverse transcribed from total RNA samples using small RNA-specific, stem-loop RT primer from the TaqMan small RNA Assays and reagents from the TaqMan microRNA reverse transcription kit. MiR-195 levels were then detected using a TaqMan microRNA kit (Applied Biosystems) and normalized to U6 snRNA. The relative amount of miRNA was calculated using $\triangle \mathrm{Ct}$. The Assay ID of miR-195 probe and U6 snRNA probe provided by ABI (USA) were 000494 and 001973, respectively. The sequences used in this work were listed as follows: AKT3 forward primer, 5'-TGAAGTGGCACACACTCTAACT-3'; AKT3 reverse primer, 5'-CCGCTCTCTCGACAAATGGA-3'; U6 RT primer, 5'-AAAATATGGAACGCT TCACGAATTTGG-3'; U6 forward primer, 5'-CTCGCTTCGGCAGCACATATACT-3'; U6 reverse primer, 5'-ACGCTTCACGAATTTGCGTGTC-3'. Each reaction was performed three times, independently.

\section{Luciferase reporter assay}

Luciferase reporter assay was performed in $293 \mathrm{~T}$ cells to confirm whether AKT3 was a direct target of miR-195. The 3'-untranslated regions (3'-UTR) of AKT3 containing the miR-195 binding site was cloned into the downstream of luciferase gene to generate pMIR-AKT3WT vector, and the 3'-UTR without miR-195 binding site was used to construct pMIR-AKT3-Mut vector. Afterwards, the pRL-TK vector, pMIR-AKT3-WT vector or pMIR-AKT3-Mut vector were in the combination of miR-195 mimic or Scr to co-transfect 293 T cells. The pGL3 vector group was served as control. Transfected cells were seeded into 24-well culture plate. After $48 \mathrm{~h}$ of transfection, luciferase activity was detected using the Dual Luciferase Reporter Assay System (Qiagen, Germany).

\section{Western blot}

Cells were collected after $24 \mathrm{~h}$ of transfection, and total cells were washed thrice with phosphate-buffered saline $(1 \times \mathrm{PBS})$. The addition of $100 \mu \mathrm{l}$ lysis buffer in each well was to obtain total cell lysates and the process of lysis was performed on ice for $30 \mathrm{~min}$. The sample was then centrifuged for $10 \mathrm{~min}$ at $12000 \mathrm{rpm}$. The supernatant was removed and its protein concentration was measured according to the BCA kit instructions. Total cellular proteins were isolated and loaded using 10\% SDS-PAGE. The primary antibodies (AKT3, 1:1000, Abcam, England; GAPDH, 1:1000, Abcam, England) were incubated at $4^{\circ} \mathrm{C}$ overnight. Afterwards, AKT3 was incubated with the goat anti-rabbit $\operatorname{IgG}(1: 10000$, Abcam, England) labeled with horseradish peroxidase, and GAPDH was with goat anti-mouse IgG (1:10000, Abcam, England) labeled with horseradish peroxidase for one hour at room temperature. Immunoreactivity was measured by an ECL kit (Amersham, Sweden).

\section{Cell migration assays}

Scratch wound-healing assays were carried out to examine GC cell migration. After $24 \mathrm{~h}$ of post-transfection, cells were plated into 12-well culture plate to achieve a confluent monolayer. Then, uniform wounds were scraped with a sterile $200 \mu \mathrm{l}$ pipette tip in 12-well culture plate, and each well was washed thrice with $1 \times$ PBS to remove floating cells. The initial distance $(0 \mathrm{~h})$ and the residual distance $(24,48$ and $72 \mathrm{~h}$ after scratch) were inspected microscopically.

\section{Cell proliferation assays}

CCK-8 was conducted to detect the cell proliferative properties of transfected cells according to the manufacturer's recommendations (Dojindo Inc., Kumamoto, Japan). Transfected cells were seeded into 96-well culture plates with $100 \mu \mathrm{l}$ culture medium. At a regular interval $(0,12,24,36$ and $48 \mathrm{~h})$ of treatment with cisplatin (DDP), each well was added $10 \mu \mathrm{lCCK}$ 8 solution, respectively. After $2 \mathrm{~h}$ of further incubation with CCK-8 solution, the visual density of each well was measured at $450 \mathrm{~nm}$ wavelength by MK3 Microplate Reader (Bio-Rad, USA), and the relative number of optical cells in each well was counted.

\section{Cell invasion assays}

Transwell assays were used to evaluate invasive abilities of GC cells in vitro. Cells were transferred into the upper wells of 24-well transwell matrigel chambers 
(BD Biosciences, USA) after $48 \mathrm{~h}$ of transfection. DMEM medium supplemented with $10 \%$ fetal bovine serum served as chemoattractant filling in the lower wells. After $48 \mathrm{~h}$ of incubation, non-invading cells on the upper surface of membranes were removed, and cells that had invaded to the lower surface of membranes were stained with Giemsa for $30 \mathrm{~min}$ and calculated under a light microscope in ten random fields.

\section{In vivo intratumoral delivery of miR-195}

MGC-803 cells $\left(1 \times 10^{6}\right)$ were resuspended in $100 \mu \mathrm{L}$ of PBS and implanted into the nude mice. Once palpable tumors (average volume of $62.5 \mathrm{~mm}^{3}$ ) were detected (usually on the $10^{\text {th }}$ day), 2 nmol of miR-195 agomir or $\mathrm{NC}$ was diluted into $25 \mu \mathrm{l}$ of autoclaving sterilized PBS, and then the mixture solution was intratumorally injected into nude mice $(n=5)$ through muti-point injection at 3-day interval. A total of four interval injections were conducted. Tumors were measured using a digital caliper. The equation of calculating tumor volumes calculated was as follows: $V\left(\mathrm{~mm}^{3}\right)=L \times P^{2} / 2$, where $L$ was the largest diameter and $P$ was the perpendicular diameter. In 26 days after administration, mice were euthanized and tumor tissues were collected for continuous experiments. All animal care was in accordance with institutional guidelines.

\section{MiRNA microarray analysis}

The tumor tissues and adjacent normal tissues of two GC patients were collected for the microarray analysis. Microarray analyses were performed by Bioassay Laboratory of CapitalBio Corporation (Beijing, China). RNA extraction, the purification and dephosphorylation intersected with labeling reaction of total RNA were all performed according to the manufacturer's instructions. In the whole process, labeling spike-in RNA and Hyb spike-in RNA (Agilent Technologies, Inc., Santa Clara, CA, USA) were added and used as quality control. Microarray slides were scanned and microarray images were automatically analyzed using Feature extraction ${ }^{\mathrm{TM}}$ software, version 10.7 (Agilent Technology, Inc., Santa Clara, CA, USA). GeneSpring (Agilent Technologies, Inc., Santa Clara, CA, USA) was used to normalize the data and perform variation analysis. Differentially expressed miRNAs were screened out according to fold change (FC) $>2$ and the related heat maps were drawn based on the tools reported by Deng W, et al [38].

\section{Statistical analysis}

Each experiment was repeated three times, and all values were presented as the means \pm standard deviation (SD). Unless otherwise indicated, T-test and ANOVA was employed respectively to analyze the difference in two groups and multiple groups $(>2)$. Chi-square test was used for analyzing the classification variables between different groups. Kaplan-Meier and log-rank test were used to perform survival analysis. SPSS version 18.0 (SPSS, Chicago, IL) was applied to complete statistical analysis. Value of $P<0.05$ was considered statistically significant for all statistical analyses.

\section{Abbreviations}

microRNAs (miRNAs); gastric cancer (GC); phosphoinositide 3-kinase (PI3K); real-time quantitative polymerase chain reaction (RT-qPCR); scramble (Scr); negative control (NC); Cell Counting Kit-8 (CCK-8); cisplatin (DDP); tumor node metastasis (TNM); overall survival (OS); progression free survival (PFS); median survival time (MST); vascular endothelial growth factor (VEGF); phosphate-buffered saline (PBS); Dulbecco's Modified Eagle's Medium (DMEM); standard deviation (SD).

\section{ACKNOWLEDGMENTS}

This study was supported by the Subject Assignment of China National Key Research and Development Programme (2016YFC0105715) and the General Program of National Natural Science Foundation of China (81402539; 81301781).

\section{CONFLICTS OF INTEREST}

The authors declare no conflicts of interest.

\section{REFERENCES}

1. Shin VY, Chu KM. MiRNA as potential biomarkers and therapeutic targets for gastric cancer. World J Gastroenterol. 2014; 20: 10432-9.

2. Rukov JL, Wilentzik R, Jaffe I, Vinther J, Shomron N. Pharmaco-miR: linking microRNAs and drug effects. Brief Bioinform. 2014; 15: 648-59.

3. Bushati N, Cohen SM. microRNA functions. Annu Rev Cell Dev Biol. 2007; 23: 175-205.

4. Hainaut P, Plymoth A. Targeting the hallmarks of cancer: towards a rational approach to next-generation cancer therapy. Curr Opin Oncol. 2013; 25: 50-1.

5. Macfarlane LA, Murphy PR. MicroRNA: Biogenesis, Function and Role in Cancer. Curr Genomics. 2010; 11: 537-61.

6. Deng H, Guo Y, Song H, Xiao B, Sun W, Liu Z, Yu X, Xia T, Cui L, Guo J. MicroRNA-195 and microRNA-378 mediate tumor growth suppression by epigenetical regulation in gastric cancer. Gene. 2013; 518: 351-9.

7. Wang Y, Zhang X, Zou C, Kung HF, Lin MC, Dress A, Wardle F, Jiang BH, Lai L. miR-195 inhibits tumor growth 
and angiogenesis through modulating IRS1 in breast cancer. Biomed Pharmacother. 2016; 80: 95-101.

8. Li Z, Wang H, Wang Z, Cai H. MiR-195 inhibits the proliferation of human cervical cancer cells by directly targeting cyclin D1. Tumour Biol. 2016; 37: 6457-63.

9. Zhang C, Liu K, Li T, Fang J, Ding Y, Sun L, Tu T, Jiang X, Du S, Hu J, Zhu W, Chen H, Sun X. miR-21: A gene of dual regulation in breast cancer. Int J Oncol. 2016; 48: 161-72.

10. Faltejskova P, Besse A, Sevcikova S, Kubiczkova L, Svoboda M, Smarda J, Kiss I, Vyzula R, Slaby O. Clinical correlations of miR-21 expression in colorectal cancer patients and effects of its inhibition on DLD1 colon cancer cells. Int J Colorectal Dis. 2012; 27: 1401-8.

11. Li B, Ren S, Li X, Wang Y, Garfield D, Zhou S, Chen X, Su C, Chen M, Kuang P, Gao G, He Y, Fan L, et al. MiR21 overexpression is associated with acquired resistance of EGFR-TKI in non-small cell lung cancer. Lung Cancer. 2014; 83: 146-53.

12. Yang SM, Huang C, Li XF, Yu MZ, He Y, Li J. miR-21 confers cisplatin resistance in gastric cancer cells by regulating PTEN. Toxicology. 2013; 306: 162-8.

13. Siegel RL, Miller KD, Jemal A. Cancer statistics, 2016. CA Cancer J Clin. 2016; 66: 7-30.

14. Orditura M, Galizia G, Sforza V, Gambardella V, Fabozzi A, Laterza MM, Andreozzi F, Ventriglia J, Savastano B, Mabilia A, Lieto E, Ciardiello F, De Vita F. Treatment of gastric cancer. World J Gastroenterol. 2014; 20: 1635-49.

15. Shi Y, Zhou Y. The role of surgery in the treatment of gastric cancer. J Surg Oncol. 2010; 101: 687-92.

16. Marin JJ, Al-Abdulla R, Lozano E, Briz O, Bujanda L, Banales JM, Macias RI. Mechanisms of Resistance to Chemotherapy in Gastric Cancer. Anticancer Agents Med Chem. 2016; 16: 318-34.

17. Sun M, Wang G, Paciga JE, Feldman RI, Yuan ZQ, Ma XL, Shelley SA, Jove R, Tsichlis PN, Nicosia SV, Cheng JQ. AKT1/PKBalpha kinase is frequently elevated in human cancers and its constitutive activation is required for oncogenic transformation in NIH3T3 cells. Am J Pathol. 2001; 159: 431-7.

18. Zhou X, Tan M, Stone Hawthorne V, Klos KS, Lan KH, Yang Y, Yang W, Smith TL, Shi D, Yu D. Activation of the Akt/mammalian target of rapamycin/4E-BP1 pathway by ErbB2 overexpression predicts tumor progression in breast cancers. Clin Cancer Res. 2004; 10: 6779-88.

19. Izuishi K, Kato K, Ogura $T$, Kinoshita $T$, Esumi $H$. Remarkable tolerance of tumor cells to nutrient deprivation: possible new biochemical target for cancer therapy. Cancer Res. 2000; 60: 6201-7.

20. Linnerth-Petrik NM, Santry LA, Moorehead R, Jücker M, Wootton SK, Petrik J. Akt isoform specific effects in ovarian cancer progression. Oncotarget. 2016; 7: 74820-33. https://doi.org/10.18632/oncotarget.11204.

21. Paul-Samojedny M, Pudelko A, Kowalczyk M, FilaDanilow A, Suchanek-Raif R, Borkowska P, Kowalski J.
Combination Therapy with AKT3 and PI3KCA siRNA Enhances the Antitumor Effect of Temozolomide and Carmustine in T98G Glioblastoma Multiforme Cells. BioDrugs. 2016; 30: 129-44.

22. Grottke A, Ewald F, Lange T, Norz D, Herzberger C, Bach J, Grabinski N, Graser L, Hoppner F, Nashan B, Schumacher U, Jucker M. Downregulation of AKT3 Increases Migration and Metastasis in Triple Negative Breast Cancer Cells by Upregulating S100A4. PLoS One. 2016; 11: e0146370.

23. Wang Q, Yu WN, Chen X, Peng XD, Jeon SM, Birnbaum MJ, Guzman G, Hay N. Spontaneous Hepatocellular Carcinoma after the Combined Deletion of Akt Isoforms. Cancer Cell. 2016; 29: 523-35.

24. Calin GA, Sevignani C, Dumitru CD, Hyslop T, Noch E, Yendamuri S, Shimizu M, Rattan S, Bullrich F, Negrini M, Croce CM. Human microRNA genes are frequently located at fragile sites and genomic regions involved in cancers. Proc Natl Acad Sci U S A. 2004; 101: 2999-3004.

25. Markou A, Tsaroucha EG, Kaklamanis L, Fotinou M, Georgoulias V, Lianidou ES. Prognostic value of mature microRNA-21 and microRNA-205 overexpression in nonsmall cell lung cancer by quantitative real-time RT-PCR. Clin Chem. 2008; 54: 1696-704.

26. Zhu S, Si ML, Wu H, Mo YY. MicroRNA-21 targets the tumor suppressor gene tropomyosin 1 (TPM1). J Biol Chem. 2007; 282: 14328-36.

27. Frankel LB, Christoffersen NR, Jacobsen A, Lindow M, Krogh A, Lund AH. Programmed cell death 4 (PDCD4) is an important functional target of the microRNA miR-21 in breast cancer cells. J Biol Chem. 2008; 283: 1026-33.

28. Wang R, Zhao N, Li S, Fang JH, Chen MX, Yang J, Jia WH, Yuan Y, Zhuang SM. MicroRNA-195 suppresses angiogenesis and metastasis of hepatocellular carcinoma by inhibiting the expression of VEGF, VAV2, and CDC42. Hepatology. 2013; 58: 642-53.

29. Liu L, Chen L, Xu Y, Li R, Du X. microRNA-195 promotes apoptosis and suppresses tumorigenicity of human colorectal cancer cells. Biochem Biophys Res Commun. 2010; 400: 236-40.

30. Yongchun Z, Linwei T, Xicai W, Lianhua Y, Guangqiang Z, Ming Y, Guanjian L, Yujie L, Yunchao H. MicroRNA-195 inhibits non-small cell lung cancer cell proliferation, migration and invasion by targeting MYB. Cancer Lett. 2014; 347: 65-74.

31. Yang G, Wu D, Zhu J, Jiang O, Shi Q, Tian J, Weng Y. Upregulation of miR-195 increases the sensitivity of breast cancer cells to Adriamycin treatment through inhibition of Raf-1. Oncol Rep. 2013; 30: 877-89.

32. Shen YH, Xie ZB, Yue AM, Wei QD, Zhao HF, Yin HD, Mai W, Zhong XG, Huang SR. Expression level of microRNA-195 in the serum of patients with gastric cancer and its relationship with the clinicopathological staging of the cancer. Eur Rev Med Pharmacol Sci. 2016; 20: 1283-7.

33. Martelli AM, Nyakern M, Tabellini G, Bortul R, Tazzari PL, Evangelisti C, Cocco L. Phosphoinositide 3-kinase/ 
Akt signaling pathway and its therapeutical implications for human acute myeloid leukemia. Leukemia. 2006; 20: 911-28.

34. Ahmad A, Biersack B, Li Y, Kong D, Bao B, Schobert R, Padhye SB, Sarkar FH. Targeted regulation of PI3K/ Akt/mTOR/NF-kappaB signaling by indole compounds and their derivatives: mechanistic details and biological implications for cancer therapy. Anticancer Agents Med Chem. 2013; 13: 1002-13.

35. Qu J, Zhao L, Zhang P, Wang J, Xu N, Mi W, Jiang X, Zhang C, Qu J. MicroRNA-195 chemosensitizes colon cancer cells to the chemotherapeutic drug doxorubicin by targeting the first binding site of BCL2L2 mRNA. J Cell Physiol. 2015; 230: 535-45.
36. Yang X, Yin J, Yu J, Xiang Q, Liu Y, Tang S, Liao D, Zhu B, Zu X, Tang H, Lei X. miRNA-195 sensitizes human hepatocellular carcinoma cells to 5-FU by targeting BCLw. Oncol Rep. 2012; 27: 250-7.

37. Yan B, Guo Q, Nan XX, Wang Z, Yin Z, Yi L, Wei YB, Gao YL, Zhou KQ, Yang JR. Micro-ribonucleic acid 29b inhibits cell proliferation and invasion and enhances cell apoptosis and chemotherapy effects of cisplatin via targeting of DNMT3b and AKT3 in prostate cancer. Onco Targets Ther. 2015; 8: 557-65.

38. Deng W, Wang Y, Liu Z, Cheng H, Xue Y. HemI: A Toolkit for Illustrating Heatmaps. PLoS One. 2014; 9: e111988. 